\title{
Martial Arts Training Prediction Model Based on Big Data and MEMS Sensors
}

\author{
Shisen Li, ${ }^{1}$ Chao Liu $\mathbb{D}^{2},{ }^{2}$ and Guoliang Yuan ${ }^{2}$ \\ ${ }^{1}$ Department of Physical Education, North China University of Science and Technology, Tangshan 063210, Hebei, China \\ ${ }^{2}$ College of Physical Education, Heng Shui University, Heng Shui 053000, Hebei, China \\ Correspondence should be addressed to Chao Liu; liuchaotrea@163.com
}

Received 9 March 2021; Revised 20 April 2021; Accepted 3 May 2021; Published 27 May 2021

Academic Editor: Shaukat Ali

Copyright (c) 2021 Shisen Li et al. This is an open access article distributed under the Creative Commons Attribution License, which permits unrestricted use, distribution, and reproduction in any medium, provided the original work is properly cited.

In martial arts teaching and sports training, the accurate capturing and analysis of martial arts athletes' posture is conducive to accurately judge sports postures, as well as correcting sports movements in a targeted manner, further improving martial arts athletes' performance and reducing physical damage. The manufacturing level of MEMS sensors continues to improve, and status perception of assembly objects is becoming more and more abundant and accurate. The shape is small and can be worn, and data can be collected continuously without obstacles. The price is relatively low, the privacy protection is strong, and the advantages are clear and prominent. A considerable number of technicians choose to use MEMS sensors as the main tool for human behavior detection data collection. Therefore, this article designs multiple MEMS inertial sensors to form a human body lower limb capture device, and its core components are composed of accelerometer, gyroscope, and magnetometer. In order to make the obtained acceleration value, angular velocity value, and magnetometer value accurately reflect the movement state of the lower limb structure, different data fusion algorithms and magnetometer ellipsoid fitting calibration algorithms are studied to realize the calculation of the posture angle of each joint point and obtain martial arts posture big data. In addition, through big data analysis, this article designs a martial arts training performance and injury risk prediction model, which can provide guidance and suggestions for martial arts teaching tasks.

\section{Introduction}

In martial arts teaching and sports training, the accurate capturing and analysis of martial arts athletes' posture is conducive to accurately judging sports postures, as well as correcting sports movements in a targeted manner, further improving martial arts athletes' performance and reducing physical damage. At present, human motion posture capture technology is mainly based on three methods: visual images [1-4], electromyographic signals [5-7], and wearable inertial sensors [8-10]. Among them, the human body gesture capture technology based on wearable inertial sensors has the advantages of directness, reliability, and strong applicability.

The mainstream human motion capture systems are optical and MEMS inertial sensors [11]. The optical motion capture system is currently the most authoritative motion capture method. Its capture range is wide, the response speed is fast, and the data delay is small. It can realize the real-time capture of the action. However, the data processing process of the optical work capture system includes identification, tracking, and coordinate calculation. The calculation workload is relatively large, the related equipment is expensive, the optical conditions are demanding, and the effect of motion capture in the occluded state is poor. The MEMS-type inertial sensor motion capture analysis equipment can overcome the problem of insufficient test accuracy of the optical system in the low-light environment and motion blocking state, and the cost of the equipment is low, the test accuracy is high, and the response speed is fast, which is conducive to real-time motion capture. At the same time, it can support the parallel capture of the motion states of multiple objects.

In recent years, the concept of "big data" has gradually attracted attention. Its definition originated from the explosion of massive data in the information age, and it was defined as the 
technological development and innovation related to it. Martial arts $[12,13]$ is one of the latest sports projects to apply the concept of big data. In today's highly developed professional events, with the advancement of science and technology and the update of equipment, the data that can be collected is more and more abundant, not only for the audience. It provides the possibility of in-depth understanding of the superb skills of toplevel martial arts athletes and also provides data support for martial arts athletes to improve their competitive skills and formulate training competition programs.

Therefore, this article designs multiple MEMS inertial sensors to form a human body lower limb capture device, and its core components are composed of accelerometer, gyroscope, and magnetometer. In order to make the obtained acceleration value, angular velocity value, and magnetometer value accurately reflect the movement state of the lower limb structure, different data fusion algorithms and magnetometer ellipsoid fitting calibration algorithms are studied to realize the calculation of the posture angle of each joint point and obtain martial arts posture big data. In addition, through big data analysis, this paper designs a martial arts training performance and injury risk prediction model, which can provide guidance and suggestions for martial arts teaching tasks. The following are the main contribution points of this paper:

(i) Aiming at the analysis of martial arts movement posture, this paper designs multiple MEMS inertial sensors to form a human lower limb capture device. Its core components are composed of accelerometer, gyroscope, and magnetometer. In order to make the obtained acceleration value, angular velocity value, and magnetometer value accurately reflect the movement state of the lower limb structure, different data fusion algorithms and magnetometer ellipsoid fitting and calibration algorithms are also studied, and the posture angle of each joint point is calculated.

(ii) Aiming at martial arts training performance and injury risk prediction, this paper proposes a big data analysis method and designs a support vector regression model to achieve more accurate prediction.

(iii) This paper also conducted a simulation experiment. Experimental results show that the algorithm can effectively improve the accuracy of martial arts training performance prediction and injury risk prediction.

The remainder of the paper is organized as follows Section 2 briefly describes the background of the study. Section 3 depicts the methodology of the proposed study. The experimental work is done in Section 4, and the paper is concluded in Section 5.

\section{Background}

There is a wearable inertial sensor product in the market as shown in Figure 1, but it has the disadvantages of large size and troublesome wearing. Some scholars fix the acceleration

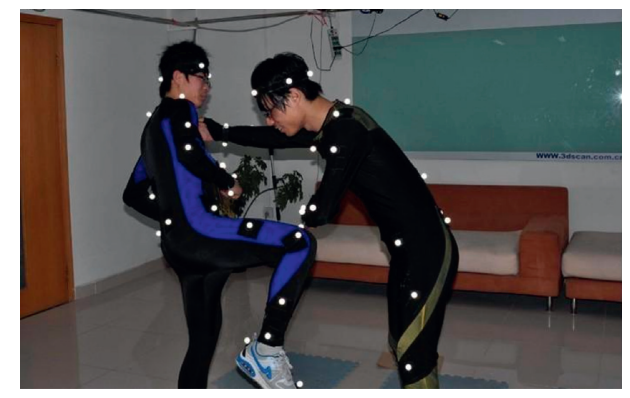

Figure 1: Martial arts motion capture system based on MEMS sensor.

sensor on the human body's motion part and realize the measurement of 5 common motion postures of the human body. However, the sensitivity of the acceleration sensor used is not high enough to recognize subtle movements. The accelerometer is worn by the athlete to measure the athlete's motion posture, thereby identifying high-risk events of sports injury in the game, but the frequency sampling range of the accelerometer needs to be improved, and the capture of more rapidly changing movements is limited. In the process of human body posture capture, the accelerometer is also required to have the characteristics of small size, low power consumption, and being easy to wear. Therefore, the study of MEMS accelerometers [14, 15] with high sensitivity and high frequency response is of great significance for human body posture capture.

2.1. MEMS Inertial Sensor. The emergence of Microelectromechanical Systems (MEMS) technology has led to a huge revolution in martial arts posture measurement technology, making martial arts posture measurement develop in the direction of low cost, low power consumption, and miniaturization. The combination of MEMS accelerometer and MEMS gyroscope is generally called an inertial measurement unit, which is used for the measurement and analysis of object posture [16]. In this paper, MEMS sensors are used to analyze the gait characteristics of martial arts athletes. In order to improve the measurement accuracy, based on the use of MEMS accelerometers and MEMS gyroscopes, magnetometers are introduced to correct the posture parameters such as acceleration and angular velocity obtained from the test to further improve the accuracy of gait parameters. Obtain and provide powerful experimental data support for gait feature analysis. The working principles, structural features, and uses of MEMS accelerometers, MEMS gyroscopes, and MEMS magnetometers are introduced below. On this basis, a sensor system suitable for motion capture in this research is selected.

2.1.1. MEMS Gyroscope. According to different components, gyroscopes can be divided into mechanical gyroscopes [16], fiber optic gyroscopes [17], laser gyroscopes [18], MEMS gyroscopes, and so forth. The mechanical gyroscope is a mechanical device that uses the gyro torque generated by the vibration of the carrier to measure the 
angular movement. It has extremely high requirements on the processing technology, the structure is very complicated, and the accuracy is restricted in many aspects. The fiber optic gyroscope (FOG) uses the Sagnac effect to sense the change of direction, thereby realizing the function of a mechanical gyroscope. Its working principle is based on light interference, with high sensitivity and stable and reliable operation. The principle of the laser gyroscope is to use an optical oscillator as an optical loop, and its distinguishing features are simple structure and long working life. MEMS gyroscope is a microelectromechanical gyroscope, which refers to a technology that uses silicon as a substrate for micron-level processing. It generally uses the principle of the angular velocity of the vibrating object sensor. MEMS gyroscopes have the characteristics of low power consumption, small size, low cost, easy installation, and mass production. Therefore, they are widely used in the low-end market. Various gyroscopes are shown in Figure 2. Due to the high cost and large volume of mechanical gyroscopes, fiber optic gyroscopes, and laser gyroscopes, they are not suitable for human wear. Therefore, this article chooses MEMS gyroscope as one of the devices in the inertial measurement unit. However, most MEMS gyroscopes have the characteristics of high noise and serious drift. To make up for this shortcoming, this article uses accelerometers and magnetometers to compensate the gyroscope's attitude measurement accuracy, which is one of the research focuses of this article. In this paper, the angular velocity provided by the gyroscope is used for data fusion with acceleration and magnetic value to calculate the attitude angle; on the other hand, the angular velocity is used as a monitoring signal for zero-speed judgment and as a signal for multisensor feature layer fusion.

2.1.2. MEMS Accelerometer. With the continuous development and maturity of MEMS acceleration sensor technology, MEMS acceleration sensors with high sensitivity, small size, and low price have been applied to electronic consumer products, such as mobile phones, smart watches, and notebook computers. The MEMS accelerometer is mainly used to measure the acceleration of the carrier. The carrier speed can be obtained by integrating the acceleration once, and the carrier displacement can be obtained by integrating again. Compared with the gyroscope, the accelerometer has good static stability, while the gyroscope has very good dynamic stability, which leads to relatively unreliable data at rest. The accelerometer can only accurately measure the attitude when the linear acceleration is negligible when stationary or in motion, while the azimuth update of the gyroscope will be affected by drift, and the data of the magnetometer is easily disturbed by the surrounding magnetic field. Therefore, this article chooses accelerometer as one of the inertial measurement unit devices. On the one hand, it is used to measure the acceleration value of the key nodes of the human body and obtain the corresponding static information. On the other hand, it can also be used to correct the drift error of the gyroscope and magnetometer to realize the human body.
2.2. Support Vector Machine. Support vector machine (SVM) [19] was proposed in 1964. Its theoretical basis follows the principle of structural risk minimization. After that, many scholars have studied the solution method of SVM. In 1992, Boser et al. [20] researched and proposed a block algorithm for solving SVM, which decomposed the original solution problem into a series of subproblems to be optimized and reduced the difficulty of solving calculation. In 1997, Osuna [21] proposed the use of a decomposition algorithm to solve the SVM. While ensuring that the subproblem remains unchanged, the variable that needs to be optimized is called the working set, and other nonworking set variables remain unchanged. The shortcomings of the problem size in the algorithm are too large. In 2000, Mangasarian and Musicant [22] introduced the smoothing function into the SVM solution algorithm and transformed the original constraint problem into an unconstrained problem and then used Newton's iteration method to solve it. Mangasarian and Musicant [23] also proposed the Lagrangian SVM algorithm, which converges linearly and uses the implicit Lagrangian formula to get the iterative formula of LSVM, which is more convenient to implement. Although scholars and researchers have done a lot of research on SVM solving algorithms and have achieved good results, designing more efficient SVM solving algorithms is helpful to the application and development of SVM, which is still a direction worth studying.

\section{Methodology}

3.1. Martial Arts Motion Capture Device Based on MEMS Inertial Sensor Unit. The main chip of the sensor unit constructed in this paper adopts TDK IIM-46230, and its output parameters include the acceleration value, angular velocity value, and magnetic force value of the carrier, which satisfy the research of the attitude fitting algorithm in this paper. The sensor package structure is shown in Figure 3, and the parameter index of the inertial sensor is shown in Table 1.

In the inertial sensor unit, the main performance indicators of the gyroscope include measurement range, resolution, zero bias, and zero bias stability. The zero bias is an important parameter of the gyroscope, so only its zero bias output is tested. The accelerometer test mainly measures the modulus value of its XYZ axis, and the modulus value should be equal to the local gravitational acceleration at rest. The measurement of the magnetometer also measures the modulus of its XYZ axis. Place the inertial sensor in an environment without magnetic field interference for 1 hour, and collect its output data of angular velocity, acceleration, and magnetic force.

3.2. Composition of Martial Arts Posture Analysis System Based on MEMS Inertial Sensor. The martial arts athlete's posture analysis system is mainly composed of inertial sensors, data forwarding routers, and computer terminals. The form of its composition is called the human body sensor network (BNS). Specifically, it refers to the human-centered 


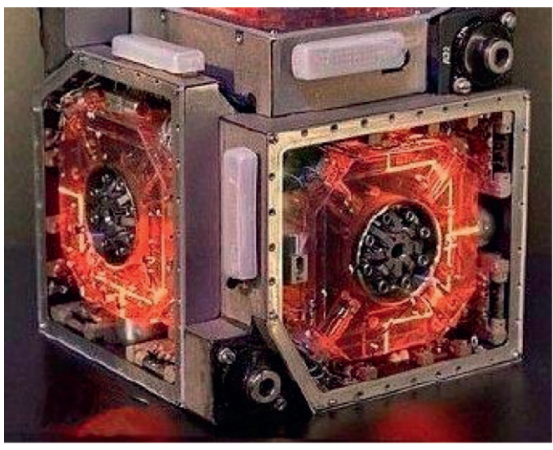

(a)

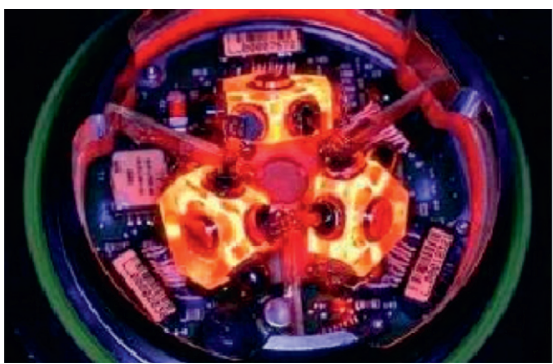

(c)

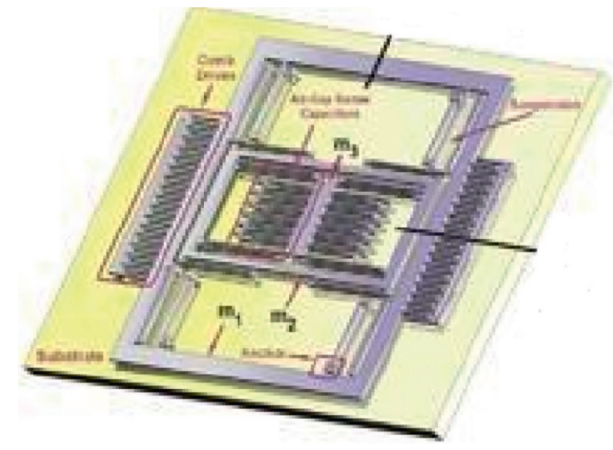

(b)

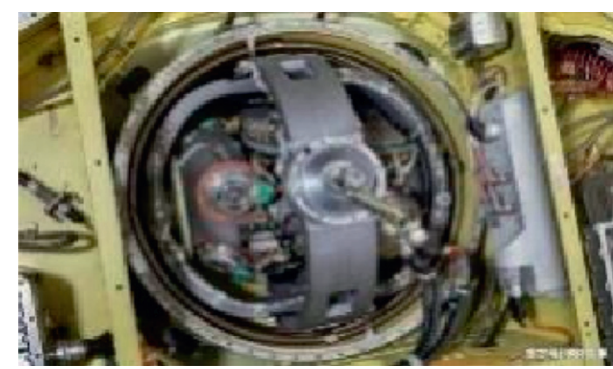

(d)

FIgURe 2: Four different kinds of gyroscopes. (a) Laser gyroscope. (b) MEMS gyroscope. (c) Fiber optic gyroscope. (d) Mechanical gyroscope.

integration of intelligent sensors, biomedicine, mechatronics, data acquisition preprocessing, multisensor data fusion, artificial intelligence, wireless communication, and other forms of multidisciplinary knowledge to realize the collection and fusion of various human motion data.

Calculation. The 7 inertial sensors of the lower limbs of martial arts athletes are used as the data collection layer of the entire system. These 7 sensors are, respectively, bound to the left instep, right instep, left calf, right calf, left thigh, right thigh, and crotch of the human body to collect various parts. The acceleration, angular velocity, and magnetic value of the magnetic field are shown in Figure 4(a). The transmission rate of the inertial sensor is $60 \mathrm{~Hz}$, the indoor transmission distance is $40 \mathrm{~m}$, the outdoor transmission distance is $110 \mathrm{~m}$, and the working time is $10 \mathrm{~h}$. The network forwarding layer is a data forwarding router, and the data of the inertial sensor is forwarded to the computer terminal through the router in the form of $2.4 \mathrm{G} \mathrm{Wi-Fi}$ forwarding. The router of the data forwarding layer is shown in Figure 4(b). The data of multiple inertial sensors collected by the data forwarding router is connected to the computer terminal through a USB cable. The inertial sensor data fusion, lower limb posture fitting, calculation of gait parameters, and various algorithm research simulations in this paper are all completed on the computing terminal, as shown in Figure 4(c).

The human sensor network of the martial arts athlete's gait analysis system is divided into three levels, namely, the data collection layer, the data transmission layer, and the data fusion calculation layer, as shown in Figure 5.
3.3. Support Vector Regression Prediction Model. As the martial arts training performance and injury risk prediction problem contains a large number of complex features, the data dimensionality is very high, and the support vector regression (SVR) machine model is a commonly used model for processing high-dimensional data, which is used in largescale complex data problems There will be very good robustness and accuracy. The core of the support vector regression model is the support vector machine (SVM), which maps high-dimensional data to a linear space and classifies it by linear regression.

Support vector machine is a two-class classification model that defines the linear classifier with the largest interval in the feature space. When the kernel function is introduced, support vector machines can also solve nonlinear problems. The main idea of the support vector machine is to find an optimal hyperplane in the feature space to completely separate the positive and negative samples, as shown in Figure 6.

For a training data set on a given feature space,

$$
T=\left\{\left(x_{1}, y_{1}\right),\left(x_{2}, y_{2}\right), \ldots,\left(x_{N}, y_{N}\right)\right\},
$$

where $x_{i} \in R^{n}, y_{\mathrm{i}} \in\{+1,-1\}, x_{i}$ represents the $i$-th feature vector, and $y_{i}$ is the class label of $x_{i}$. The model can be expressed as the following convex quadratic programming problem:

$$
\begin{aligned}
& \min _{w, b, \zeta} \frac{1}{2}\|w\|^{2}+C \sum_{i=1}^{N} \zeta_{i}, \\
& \text { s.t. } \quad y_{i}\left(w x_{i}+b\right) \geq 1-\zeta_{i}, \quad i=1,2, \ldots, N,
\end{aligned}
$$




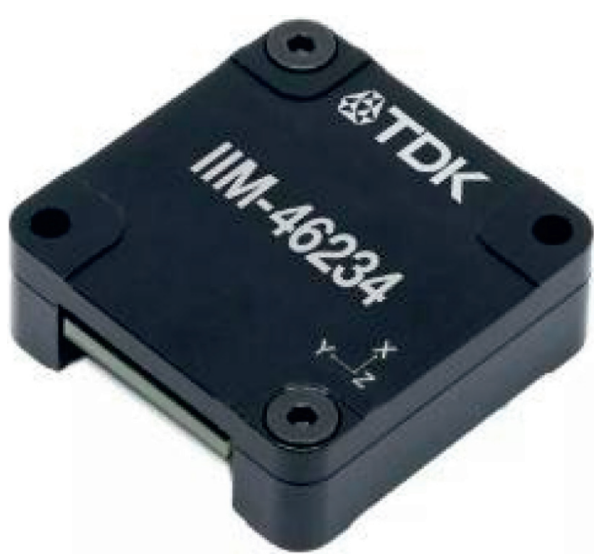

(a)

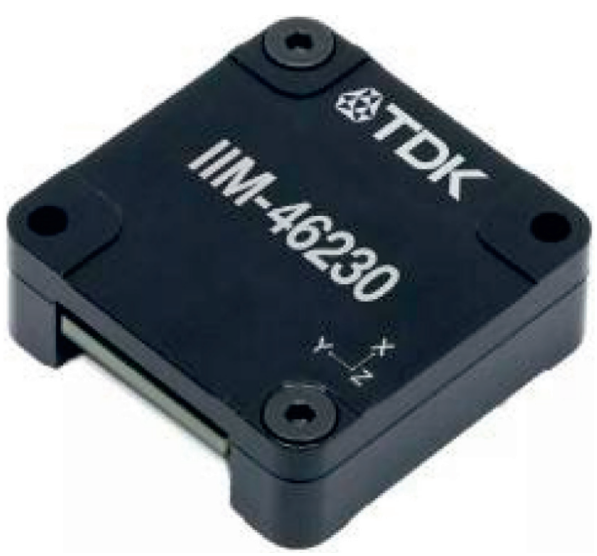

(b)

FIgURE 3: TDK IIM-46234 inertial sensor.

TABLE 1: The parameters of TDK IIM-46234 inertial sensor.

\begin{tabular}{lccc}
\hline Types & Gyro & Accelerometer & Magnetometer \\
\hline Range & $280 \% \mathrm{~s}$ & $4 \mathrm{~g}$ & $20 \mathrm{Guss}$ \\
Nonlinearity & $0.1 \%$ & $0.5 \%$ & $0.1 \% \mathrm{FS}$ \\
Noise density & 0.009 & $100 \mathrm{ug}$ & $5 \mathrm{mG}$ \\
xyz orthogonality & 0.1 & 0.1 & 1 \\
Sensitivity & $17.5 \mathrm{LSB}$ & $2048 \mathrm{LSB}$ & $0.6 \mathrm{uT} / \mathrm{LSB}$ \\
Start time & $30 \mathrm{~ms}$ & $25 \mathrm{~ms}$ & $22 \mathrm{~ms}$ \\
Output rate & $120 \mathrm{~Hz}$ & $120 \mathrm{~Hz}$ & $120 \mathrm{~Hz}$ \\
Axis & 3 -axis & 3 -axis & 3 -axis \\
\hline
\end{tabular}

$$
\zeta_{i} \geq 0, \quad i=1,2, \ldots, N
$$

where $\zeta_{i}$ is a slack variable, and $C>0$ is called a penalty parameter, which is generally determined by the actual situation. A large value of $C$ means a large penalty for misclassification, and a small value of $C$ means a small penalty for misclassification. Minimizing the objective function (equation (2)) contains two meanings: (1) Make $1 / 2\|w\|^{2}$ as small as possible; that is, maximize the interval. (2) Make the number of misclassified points as small as possible, and $C$ is a parameter that reconciles the two.

The above equations represent a convex quadratic programming problem, so the solution about WB exists; and it can be proved that the solution of $w$ is unique, but the solution of $b$ is not unique, and the solution of $b$ is in an interval. In order to solve the convex quadratic programming problem, equations (2)-(4) can be transformed into the corresponding dual problem:

$$
\begin{array}{ll}
\min & L(w, b, a)=\frac{1}{2} \sum_{i=1}^{N} \sum_{j=1}^{N} a_{i} a_{j} y_{i} y_{j}\left(x_{i} \times x_{j}\right)-\sum_{i=1}^{N} a_{i}, \\
\text { s.t. } & \sum_{i=1}^{N} a_{i} y_{i}=0, \\
& 0 \leq a_{i} \leq C, \quad i=1,2, \ldots, N,
\end{array}
$$

where $a$ is the Lagrange multiplier and $\left(x_{i} \times x_{j}\right)$ is the inner product of $x_{i}$ and $x_{j}$. The optimal solutions $w^{*}$ and $b^{*}$ of the original problem can be expressed as

$$
\begin{aligned}
& w^{*}=\sum_{i=1}^{N} a_{i}^{*} y_{i} x_{i}, \\
& b^{*}=y_{j}-\sum_{i=1}^{N} y_{i} a_{i}^{*}\left(x_{i} \times x_{j}\right) .
\end{aligned}
$$

Thus, the separating hyperplane can be expressed as

$$
\sum_{i=1}^{N} a_{i}^{*} y_{i}\left(x \times x_{i}\right)+b^{*}=0 .
$$

The classification decision function can be expressed as

$$
f(x)=\operatorname{sign}\left(\sum_{i=1}^{N} a_{i}^{*} y_{i}\left(x \times x_{i}\right)+b^{*}\right) .
$$

In the martial arts training performance and injury risk prediction, the real value is directly output:

$$
y=\sum_{i=1}^{N} a_{i}^{*} y_{i}\left(x \times x_{i}\right)+b^{*} .
$$

\section{Experiments}

4.1. Experimental Environment. The hardware platform and software simulation environment of the experiment in this paper are shown in Table 2.

4.2. AUC Evaluation Criteria. Martial arts athletes training injury risk prediction is a typical two-classification problem. It is difficult to reasonably reflect the effect of classification models that deal with large-scale data using traditional evaluation methods such as accuracy. For a two-category problem, first set a threshold according to the prediction result. When the estimated value is higher than the threshold, it means click; otherwise, it means no click. For a 


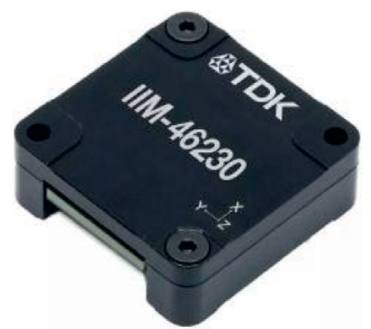

(a)

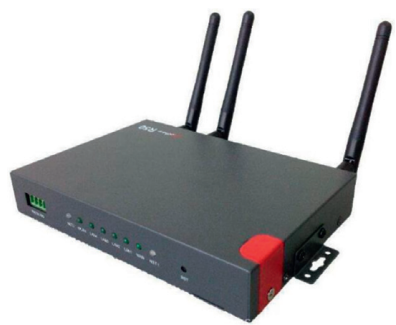

(b)

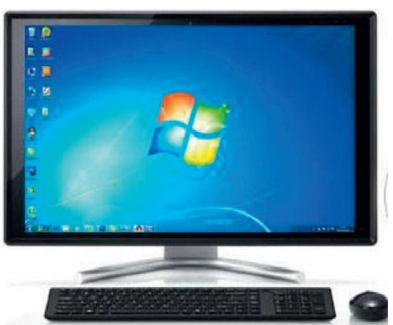

(c)

Figure 4: Physical map of martial arts athlete sensor network. (a) Data acquisition sensors. (b) Data forwarding muter. (c) Computer terminal.

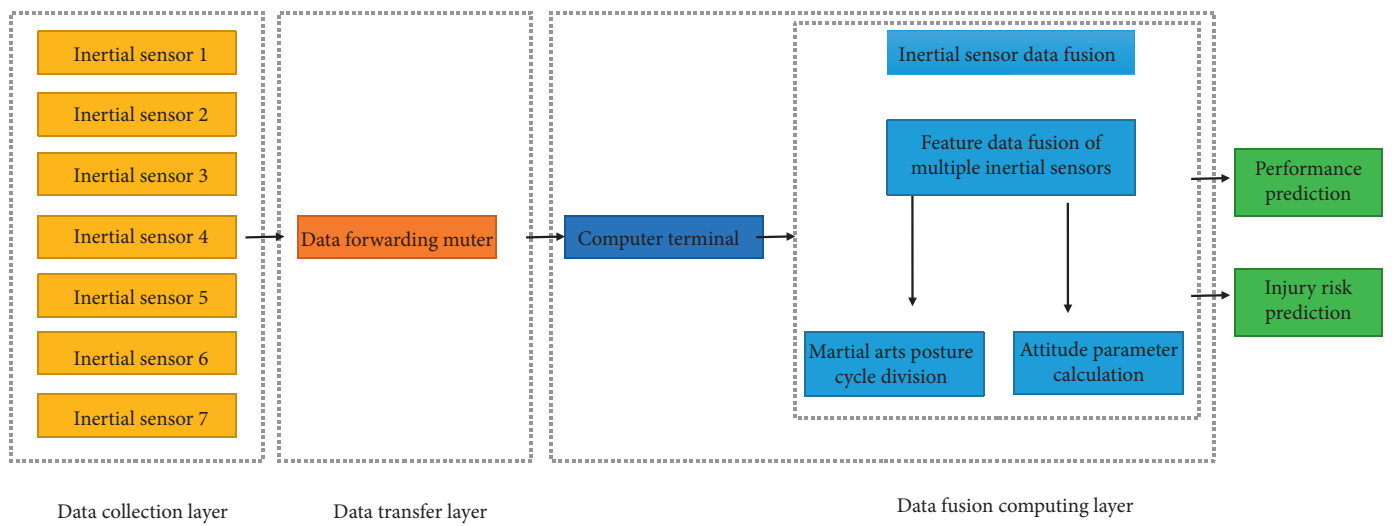

FIGURE 5: Martial arts athlete's training analysis and prediction system.

specific example, the predicted effect can be reflected by the four types in Table 3.

For the entire test set, the training injury risk prediction results of martial arts' athletes are usually described by the ROC curve. FPR is the abscissa of the curve, indicating that the risk-free records are classified into the prediction report, as shown in formula (10); the ordinate is TPR, which means that the risky records are correctly assigned to the forecast report, as shown in formula (11). Under normal circumstances, the TPR value is greater than the FPR value; otherwise, it means that the prediction result is reversed. The AUC value is used to represent the area under the curve, which is an important measurement index for the evaluation of the prediction results of the martial arts athletes' training injury wind. The higher the value, the better the forecasting effect. Moreover, the value of AUC is between 0.5 and 1 under normal circumstances, and the ROC curve is convex, as shown in Figure 7.

$$
\begin{aligned}
& \mathrm{FPR}=\frac{\mathrm{FP}}{\mathrm{FP}+\mathrm{TN}}, \\
& \mathrm{TPR}=\frac{\mathrm{TP}}{\mathrm{TP}+\mathrm{FN}} .
\end{aligned}
$$

4.3. Experimental Results and Analysis. In this paper, the training set randomly sampled from the data set is 10,000 , and the test set is 3,000. Three basic models are used to predict martial arts training performance and injury risk,

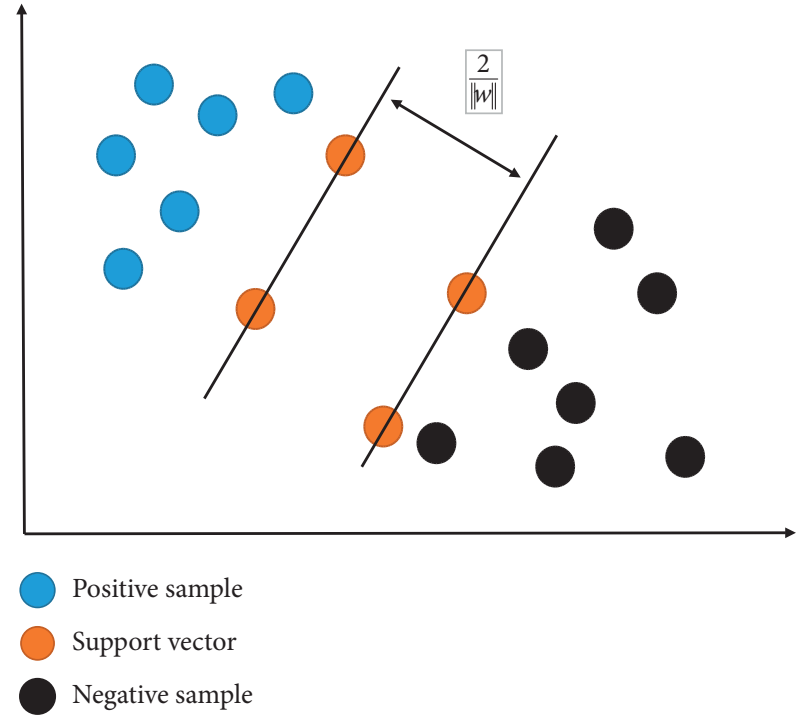

FIGURE 6: Support vector machine model.

and the AUC value and training time are used as evaluation indicators to explore the advantages and disadvantages of the logistic regression model.

This article compares the three basic models. The features extracted by the model in the experiment are shown in Table 4, where "f_Sparse" is the category sparse feature, "p_CTR" is the historical click rate feature, "Similar" is the similarity feature, "Pos" is the location feature, and "W2V" is 
TABLE 2: Experimental hardware platform and software simulation environment.

\begin{tabular}{lc}
\hline CPU & Intel(R) Core(TM) i5-2450M CPU @ 2.50 GHz (2500 MHz) \\
\hline RAM & $4.00 \mathrm{~GB}$ \\
Operating system & Ubuntu 14.04 LTS \\
Development environment & PyCharm \\
Programming language & Python \\
\hline
\end{tabular}

TABle 3: Confusion matrix.

\begin{tabular}{llc}
\hline TP & True positive & Predict the instances from the positive sample correctly \\
TN & True negetive & Predict the instances from the negative samples correctly \\
FP & False positive & Predict the instances from the positive sample incorrectly \\
FN & False negetive & Misprediction of instances from the negative samples \\
\hline
\end{tabular}

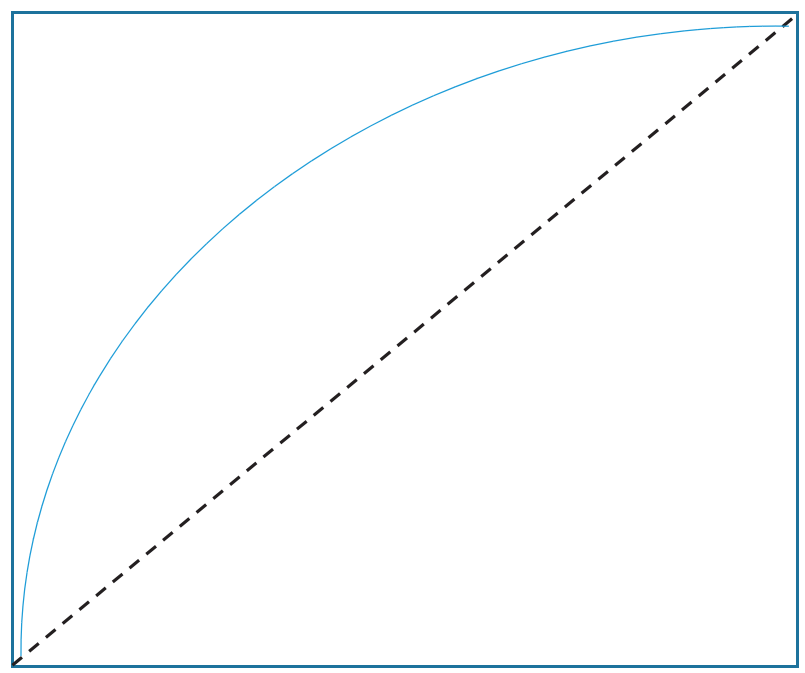

FiguRe 7: ROC curve.

TABle 4: Confusion matrix.

\begin{tabular}{llc}
\hline Model & AUC & Time (min) \\
\hline Naive Bayes & 0.642 & 18 \\
Logistic regression & 0.689 & 15 \\
Support vector regression (ours) & 0.758 & 12 \\
\hline
\end{tabular}

the word vector feature. For the case where the prior probability in the naive Bayes model is 0 , the experiment uses plus one smoothing for processing. The experimentally predicted AUC and training time are shown in Table 4.

Experiments show that the prediction effect of the naive Bayes model is obviously inferior to those of the logistic regression model and the support vector regression model. Not only is the AUC value low, but also the training time is the longest. The reason may be a hypothetical estimation based on training data in prior probability, as well as a certain correlation between features, while naive Bayes trains the model based on the assumption of feature independence. In addition, the AUC value predicted by the logistic regression model is slightly lower than that of the support vector regression model, and the training time is longer. The support vector regression model can capture the key samples in the training set and use them as support vectors, which makes the model very robust.

\section{Conclusion}

The accurate capturing and analysis of martial arts athletes' posture in martial arts teaching and sports training is beneficial to accurately judge sports postures, as well as adjusting sports movements in a targeted manner with further refining martial arts athletes' performance and decreasing physical damage. To improve the prediction accuracy of the training injury risk of martial arts athletes and reduce physical damage, this article designs multiple MEMS inertial sensors to form a human body lower limb capture device, and its core components are composed of accelerometers, gyroscopes, and magnetometers. In order to make the obtained acceleration value, angular velocity value, and magnetometer value accurately reflect the movement state of the lower limb structure, different data fusion algorithms and magnetometer ellipsoid fitting calibration algorithms are studied to realize the calculation of the posture angle of each joint point and obtain martial arts posture big data. In addition, through big data analysis, this paper designs a martial arts training performance and injury risk prediction model, which can provide guidance and suggestions for martial arts teaching tasks.

\section{Data Availability}

The data used to support the findings of this study are included within the article.

\section{Conflicts of Interest}

All the authors declare no conflicts of interest.

\section{References}

[1] L. Zhang, X. Wang, X. Dong, L. Sun, W. Cai, and X. Ning, "Finger vein image enhancement based on guided tri-gaussian filters," ASP Transactions on Pattern Recognition and Intelligent Systems, vol. 1, no. 1, pp. 17-23, 2021.

[2] W. Cai, B. Liu, Z. Wei, M. Li, and J. Kan, "TARDB-Net: tripleattention guided residual dense and BiLSTM networks for hyperspectral image classification," Multimedia Tools and Applications, vol. 80, no. 7, Article ID 11291, 2021.

[3] X. Ning, Y. Wang, W. Tian, L. I. U. Liang, and W. Cai, "A biomimetic covering learning method based on principle of 
homology continuity," ASP Transactions on Pattern Recognition and Intelligent Systems, vol. 1, no. 1, pp. 9-16, 2021.

[4] X. Ning, X. Wang, S. Xu et al., "A review of research on cotraining, concurrency and computation: practice and experience," Bio-Engineering Applications, vol. 32, Article ID e6276, 2021.

[5] H. Su, S. E. Ovur, X. Zhou, W. Qi, G. Ferrigno, and E. De Momi, "Depth vision guided hand gesture recognition using electromyographic signals," Advanced Robotics, vol. 34, no. 15 , pp. 985-997, 2020.

[6] D. Buongiorno, G. D. Cascarano, I. De Feudis et al., "Deep learning for processing electromyographic signals: a taxonomy-based survey," Neurocomputing, 2020, In press.

[7] S. Sapienza, P. M. Ros, D. A. F. Guzman et al., "On-line eventdriven hand gesture recognition based on surface electromyographic signals," in Proceedings of the 2018 IEEE International Symposium on Circuits and Systems (ISCAS), IEEE, Florence, Italy, May 2018.

[8] V. Camomilla, E. Bergamini, S. Fantozzi, and G. Vannozzi, "Trends supporting the in-field use of wearable inertial sensors for sport performance evaluation: a systematic review," Sensors, vol. 18, no. 3, p. 873, 2018.

[9] A. Ancillao, S. Tedesco, J. Barton, and B. O’Flynn, "Indirect measurement of ground reaction forces and moments by means of wearable inertial sensors: a systematic review," Sensors, vol. 18, no. 8, p. 2564, 2018.

[10] M. Guo, Z. Wang, N. Yang, Z. Li, and T. An, "A multisensor multiclassifier hierarchical fusion model based on entropy weight for human activity recognition using wearable inertial sensors," IEEE Transactions on Human-Machine Systems, vol. 49, no. 1, pp. 105-111, 2018.

[11] Z. Chao, F. Pu, Y. Yin, B. Han, and X. Chen, "Research on real-time local rainfall prediction based on MEMS sensors," Journal of Sensors, vol. 2018, Article ID 6184713, 9 pages, 2018.

[12] R. A. Fabio and G. E. Towey, "Cognitive and personality factors in the regular practice of martial arts," The Journal of Sports Medicine and Physical Fitness, vol. 58, no. 6, pp. 933943, 2017.

[13] L. Podrigalo, S. Iermakov, V. Potop et al., "Special aspects of psycho-physiological reactions of different skillfulness athletes, practicing martial arts," Journal of Physical Education and Sport, vol. 17, pp. 519-526, 2017.

[14] S. Łuczak, R. Grepl, and M. Bodnicki, "Selection of MEMS accelerometers for tilt measurements," Journal of Sensors, vol. 2017, Article ID 9796146, 2017.

[15] M. Varanis, A. Silva, A. Mereles, and R. Pederiva, "MEMS accelerometers for mechanical vibrations analysis: a comprehensive review with applications," Journal of the Brazilian Society of Mechanical Sciences and Engineering, vol. 40, no. 11, pp. 1-18, 2018.

[16] L. G. Pagani, L. Guerinoni, L. Falorni, P. Fedeli, and G. Langfelder, "Investigation of gyroscopes mechanical and electronic phase drift with $2 \mu \mathrm{rad} / \sqrt{ } \mathrm{hz}$ resolution and 12 $\mu \mathrm{rad} / \mathrm{K}$ accuracy," in Proceedings of the 2020 IEEE International Symposium on Inertial Sensors and Systems (INERTIAL), March 2020.

[17] G. A. Sanders, L. K. Strandjord, J. Wu et al., "Development of compact resonator fiber optic gyroscopes," in Proceedings of the 2017 IEEE International Symposium on Inertial Sensors and Systems (INERTIAL), IEEE, Kauai, HI, USA, March 2017.

[18] A. D. Di Virgilio, N. Beverini, G. Carelli, D. Ciampini, F. Fuso, and E. Maccioni, "Analysis of ring laser gyroscopes including laser dynamics," The European Physical Journal C, vol. 79, no. 7, pp. 1-8, 2019.
[19] W. S. Noble, "What is a support vector machine?" Nature Biotechnology, vol. 24, no. 12, pp. 1565-1567, 2006.

[20] B. E. Boser, I. M. Guyon, and V. N. Vapnik, "A training algorithm for optimal margin classifiers," in Proceedings of the 5th Annual Workshop on Computational Learning Theory, Pittsburgh, PA, USA, July 1992.

[21] E. E. Osuna, "Support vector machines: training and applications," Doctoral Dissertation, Massachusetts Institute of Technology, Cambridge, MA, USA, 1998.

[22] O. L. Mangasarian and D. R. Musicant, "Active support vector machine classification," in Proceedings of the 13th International Conference on Neural Information Processing Systems NIPS, MIT Press, Cambridge, MA, USA, April 2000.

[23] O. L. Mangasarian and D. R. Musicant, "Lagrangian support vector machines," Journal of Machine Learning Research, vol. 1, pp. 161-177, 2001. 